\title{
L-statistics based Modification of Reconstruction Algorithms for Compressive Sensing in the Presence of Impulse Noise
}

\author{
Srdjan Stanković, Irena Orović and Moeness Amin
}

\begin{abstract}
${ }^{1}$ Abstract- A modification of standard compressive sensing algorithms for sparse signal reconstruction in the presence of impulse noise is proposed. The robust solution is based on the Lestimate statistics which is used to provide appropriate initial conditions that lead to improved performance and efficient convergence of the reconstruction algorithms.
\end{abstract}

Keywords -reconstruction algorithms, Basis Pursuit, Orthogonal Matching Pursuit, robust statistics, L-statistics

\section{INTRODUCTION}

High variance impulse noise causes large errors when using standard sparse signal reconstruction algorithms [1]-[5]. The effect of impulsive noise can be mitigated by applying robust approaches. For instance, the myriad projections and nonlinear constraints based on the Lorentzian norm have been employed within the Basis Pursuit (BP) algorithm, producing improved results over the standard BP and Orthogonal Matching Pursuit (OMP) [8]. However, an efficient optimization of the Lorentzian norm requires complex parameter adjustments and exhaustive search procedures. Also, as stated in [8], the myriad projections used for signal measurements are computationally demanding compared to linear projections, since an optimization problem is solved for each projection. In [9], the $\ell_{0}$ regularized least absolute deviation regression model is combined with weighted median regression to obtain an approximate solution. This method also requires many computations, with the parameter selection (e.g., number of iterations and decaying speed) based on trial and error. In this paper, we propose a simple and effective CS-approach that combines the basic forms of reconstruction algorithms such as BP and OMP with the L-statistics. The latter is

\footnotetext{
1 S. Stanković and I. Orović are with the Faculty of Electrical Engineering, University of Montenegro, 20000 Podgorica, Montenegro. S. Stanković is currently on leave with Villanova University. M. Amin is with the CAC, Villanova University, PA, USA. Corresponding author e-mail: *irenao@ac.me*

The work by Professor Stankovic and Orovic is supported by the Montenegrin Ministry of Science. The work by Professor Amin is supported in part by ONR under grant N00014-11-1-0576.
}

incorporated in the initialization phase of the $\mathrm{BP}$ and OMP. Different from [8], no assumption of sparsity in the time domain is invoked. However, the signal is sparse in the transform domain, as it is often case in practice. In this paper, we also assume that the amplitudes of the impulse noise samples are not sufficiently large to be filtered out by myriad projections.

\section{THEORETICAL BACKGROUND}

We observe a one-dimensional signal $f$ of length $N$, which can be represented as a linear combination of the orthonormal basis vectors as [1]:

$$
f(n)=\sum_{k=0}^{N-1} x_{k} \psi_{k}(n) \text { or : } f=\Psi x,
$$

where $\Psi$ represents an orthonormal basis matrix, while $x$ represents a vector of transform domain coefficients. If the number of non-zero coefficients in $x$ is $S$, then we may say that $f$ is $\mathrm{S}$-sparse in the domain defined by $\Psi$. A set of $M$ random measurements are made through linear projections $y=\Phi f$ where $\Phi(M \mathrm{x} N)$ is the measurement matrix. Accordingly, we may write:

$$
y=\Phi \Psi x=A x,
$$

where $A$ is the thinned sensing matrix. The reconstructed signal is obtained as a solution of $M$ linear equations with $N$ unknowns. This system is under-determined and can have infinitely many solutions. Thus, optimization algorithms based on $\ell_{0}$ - norm minimization are used to search for the sparsest solution. In practice, the nearoptimal solutions based on the $\ell_{1}$ - norm minimization are commonly used:

$$
\min \|\tilde{x}\|_{\ell 1} \text { subject to } y=A \tilde{x} \text {. }
$$

The above problem can be solved, for example, by the commonly used simplex and interior point methods (e.g., primal-dual interior point method) [5], or by using greedy algorithms such as OMP that will provide an approximate solution. 


\section{Motivation}

Assume that the desired noise-free signal is sparse in a certain transform domain. The impulse noise, if transformed through a linear combination of the timedomain samples, will no longer be impulsive in nature and will infringe over all samples. This causes the sparse signal domain to be populated, rather than sparse, when considering the impulse noise. For example, consider a sinusoidal signal at the frequency $k_{0}$. The discrete Fourier transform of $f(n)$ corresponds to the delta pulse at the frequency $k=k_{0}$ :

$$
f(n)=C e^{j 2 \pi k_{0} n / N} \stackrel{F T}{\longleftarrow} F(k)=C N \delta\left(k-k_{0}\right),
$$

where $C$ denotes the signal amplitude, while $N$ is the number of samples. For the signal $f(n)$ we can state that it is sparse in the strict sense. On the other hand, for the noise represented by an impulse at the instant $n=n_{0}$, the Fourier transform is given in the form:

$$
v(n)=I \delta\left(n-n_{0}\right) \stackrel{F T}{\longleftarrow} V(k)=I e^{j 2 \pi k n_{0} / N},
$$

where $I$ denotes the impulse amplitude. Hence, it is obvious that each impulse in the time domain is transformed into a sinusoid in the frequency domain, and as such, when added to $f(n)$, compromises the signal sparseness property. Hence, generally, the data $f_{v}(n)=f(n)+v(n)$ is non-sparse in both time and frequency domains. A special case is when $I$ assumes a small value. In this case, $f_{V}(n)$ is nearly sparse, since $V(k)$ will be negligible compared to $F(k)$. Signal sparseness is likely to be lost when dealing with noise of multiple impulses. Hence, there is a need to subdue the noise contributions at the onset. That is, the initial condition of the iterative method adopted in the solutions of equation (3) should be impulse-free to ensure algorithm convergence to reasonable solutions.

\section{L-ESTIMATE FORMS OF SIGNAL RECONSTRUCTION METHODS}

In the case of impulse noise, the L-estimate form [10][12] of the initial Fourier transform domain vector $x_{0}$ is used in the optimization to provide efficient reconstruction. Hence, in the sequel, we propose the Lestimate primal-dual interior point method as well as the L-estimate version of OMP.

\subsection{L-estimate primal-dual interior point method}

In the case when $x, A$ and $y$ are real, the optimization problem given by (3) can be recast as the linear program:

$$
\begin{aligned}
& \min _{u} \sum u \quad \text { subject to } f_{1}=x-u \leq 0 \text {, } \\
& f_{2}=-x-u \leq 0, \\
& A x=y \text {, } \\
& \lambda_{1} f_{1}=0, \lambda_{2} f_{2}=0,
\end{aligned}
$$

with initial condition,

$$
\begin{gathered}
x(k)=x_{0}(k)=L_{\{y\}}=\sum_{i=0}^{M-1} a_{i} Y_{S}(i), \\
Y_{s}=\operatorname{sort}\left\{y(m) e^{-j 2 \pi m k / M}, \quad m=0, \ldots, M-1\right\}
\end{gathered}
$$

where $\sum_{i=0}^{M-1} a_{i}=1$, frequency range is $k=0, \ldots, N-1$, while $L$ is the L-estimation operator. The coefficients $a_{i}$ should be defined to provide impulse-free representation. Note that the sequence of elements $Y_{s}$ is sorted into nondecreasing order. In order to provide noise free $x_{0}$, we should omit $2 \alpha(M-2)$ of the highest amplitudes, while the mean is calculated over the rest of the values. Hence, the coefficients $a_{i}$ are defined as follows:

$$
a_{i}=\left\{\begin{array}{cc}
\frac{1}{M(1-2 \alpha)+4 \alpha}, & \text { for } i \in[0, M(1-2 \alpha)+4 \alpha] \\
0, & \text { elsewhere, }
\end{array}\right.
$$

where $M$ is even. The variable $\alpha$ takes values within the range $[0,1 / 2]$. The proper value of $\alpha$ should be chosen according to the expected amount of noisy samples. Now, the modified procedure can be summarized as:

1. Determine $x_{0}=L\{y\}$ for the known measurement vector $y$ and set $x=x_{0}$ for the first iteration

2. Set $u=u_{0}, \lambda_{1}$ and $\lambda_{2}$ (which can be set using $\mathrm{x}_{0}$, e.g.: $\quad 0.95\left|\mathrm{x}_{0}\right|+0.10 \max \left\{\left|x_{0}\right|\right\}, \quad \lambda_{1}=-1 /\left(x_{0}-u_{0}\right)$, $\left.\lambda_{2}=-1 /\left(-x_{0}-u_{0}\right)\right)$

3. Minimization problem can be observed using Lagrangian:

$$
\begin{aligned}
& \Lambda\left(x, u, v, \lambda_{1}, \lambda_{1}\right)=f(u)+v(A x-y)+\lambda_{1} f_{1}+\lambda_{2} f_{2}, \\
& \quad \text { where } v=-A\left(\lambda_{1}-\lambda_{2}\right)
\end{aligned}
$$

4. Solve a system of equations obtained by finding the first derivatives of $\Lambda$ in terms of $x, u, v, \lambda_{1}$ and $\lambda_{2}$, to compute Newton steps, $\Delta x, \Delta u$ and $\Delta v$.

5. Update the values of variables for the next iteration. The step length is calculated using backtracking line search.

\subsection{L-estimate OMP}

OMP is a greedy signal reconstruction algorithm that in each iteration searches for the maximum correlation between the measurements and the transform matrix. Thus, through the iterations it selects a certain number of transform matrix columns, where this number is defined 
by the given number of iterations. The least square optimization is performed afterwards in the subspace spanned by all previously picked columns. In the case of impulse noise, the L-estimate form of measurements vector can be obtained by calculating firstly the Lestimate of the initial transform domain vector $x=x_{0}$, as in the previously described case. Hence, the L-estimate OMP algorithms can be described as follows:

Step 1: Determine $x_{0}(k)=L\{y\},(k=0, \ldots, N-1)$, for the known measurement vector $y$,

$$
\begin{gathered}
x_{0}(k)=L\{y\}=\sum_{i=0}^{M-1} a_{i} Y_{s}(i), \\
Y_{S}=\operatorname{sort}\left\{y(m) e^{-j 2 \pi m k / M}, m=0, \ldots, M-1\right\} \\
\text { while } \vartheta=\arg \left\{\operatorname{sort}\left(y(m) e^{-j 2 \pi m k / M}, m=0, \ldots, M-1\right)\right\}
\end{gathered}
$$

Step 2: Set $y_{L}=\Theta x_{0}$ and $\Theta=\Psi(\vartheta)$, $\Psi$ is the Fourier transform matrix.

Step 3: Set the initial residual $r_{0}=y_{L}$ and $\Omega_{0}=\varnothing$.

Step 4: Find the maximum correlation column:

$$
\omega_{i}=\arg \max _{j}\left|\left\langle r_{i-1}, \Theta_{j}\right\rangle\right| .
$$

Step 5: Update the set: $\Omega_{i}=\Omega_{i-1} \cup \omega_{i}$.

Step 6: Solve the least square optimization problem:

$$
x_{i}=\arg \min _{x}\left\|r_{i-1}-\Omega_{i} x_{i-1}\right\|_{2}^{2} .
$$

Step 7: Update the residual: $r_{i}=r_{i-1}-\Omega_{i} x_{i-1}$. Step 8: Update $i=i+1$ and go to Step 3 if $i<K$.

\section{Simulation RESUltS}

\section{Example 1:}

Consider a signal which is the sum of five sinusoids corrupted by additive impulse noise:

$$
f(n)=\sum_{i=1}^{5} \sin (2 \pi k(i) n / N)+\xi(n),
$$

where $k=[25,45,80,100,176], t=[0,1, \ldots, N-1], N=1000$.

The number of observations used for the signal reconstructions is $M=300$, i.e., $30 \%$ of the total number of samples. In this example, the parameter $\alpha=0.07$ is used, which means that $16 \%$ of time samples are assumed to be corrupted by noisy pulses. The original non-noisy signal and its Fourier transform (FT) are shown in Fig 1.a and Fig 2.a, respectively, while the noisy signal and its FT are shown in Fig 1.b and Fig 2.b. The desired signal reconstructed by the standard BP primal-dual algorithm is shown in Fig 1.c, whereas its FT is shown in Fig 2.c. We can observe that the noise is spread across the frequency spectrum. Finally, the respective results obtained using the proposed algorithm are shown in Fig 1.d and Fig 2.d.

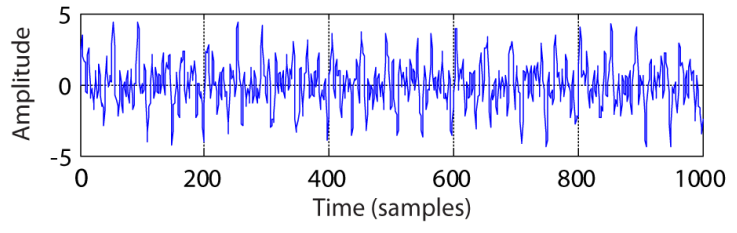

a)

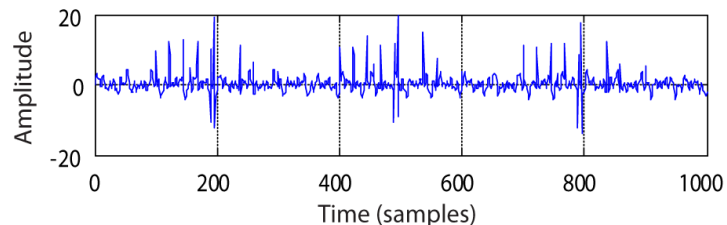

b)

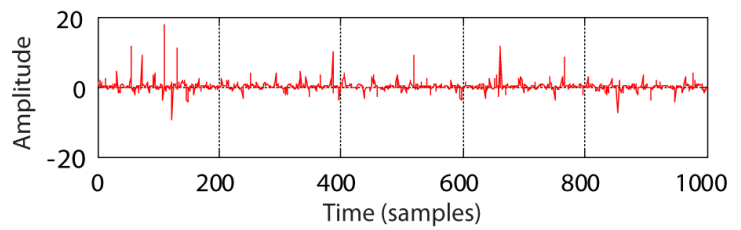

c)

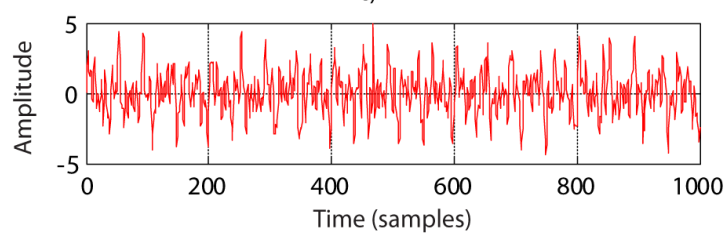

d)

Fig. 1. Time domain representations: a) Original clean signal, b) Original noisy signal, c) Reconstruction using standard primal-dual algorithm, d) Reconstruction using L-estimate primal-dual algorithm
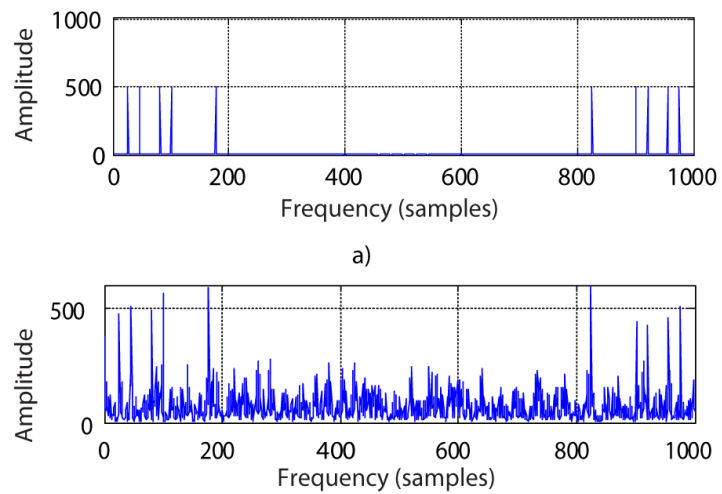

b)

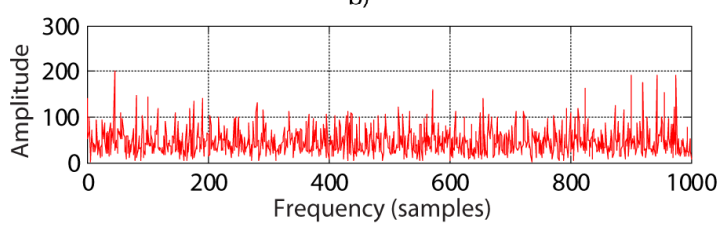

c)

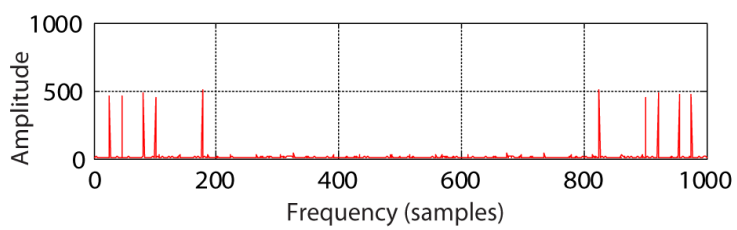

d)

Fig. 2 Fourier domain representation: a) Clean signal, b) Noisy signal, c) Reconstructed FT using standard primal-dual algorithm, d) Reconstructed FT using L-estimate primal-dual algorithm 


\section{Example 2:}

In this example, we will briefly illustrate the performance of the L-estimate OMP. Similarly as in the previous example, let us now consider a set of 10 sinusoids, defined by the set of frequencies $k=[32,80,176,267,300,350,400,560,600,680]$ and unit amplitudes. The observations used for the signal reconstructions include only $28 \%$ of the total number of signal samples. Again, parameter $\alpha=0.07$ is used ( $16 \%$ of measurements are assumed to be corrupted by strong noisy pulses). The original noise free and noisy signals are shown in Fig. 3a and b, respectively. Time domain representation of reconstructed signal using the Lestimate OMP algorithm is shown in Fig. 3c.

The original signal Fourier transform is shown in Fig 4.a, the Fourier transform obtained by applying standard OMP to the noisy signal is given in Fig 4.b, while the Fourier transform obtained by applying the proposed Lestimate OMP is shown in Fig 4.c. It is obvious that the standard OMP algorithm (Fig 4.b) fails due to the strong impulse noise, producing false components at the frequencies where the components do not appear in original signal. On the other hand, the proposed approach succeeds to recover all signal components, producing sometimes just negligible difference in amplitudes.

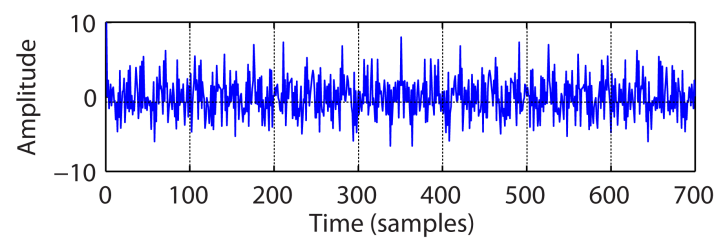

a)

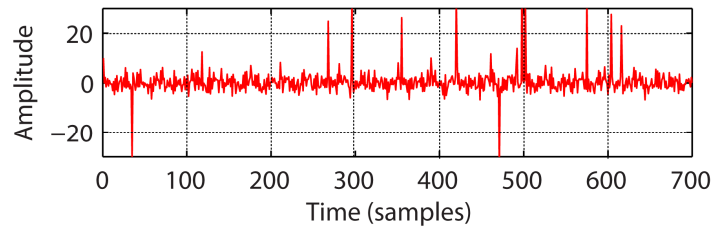

b)

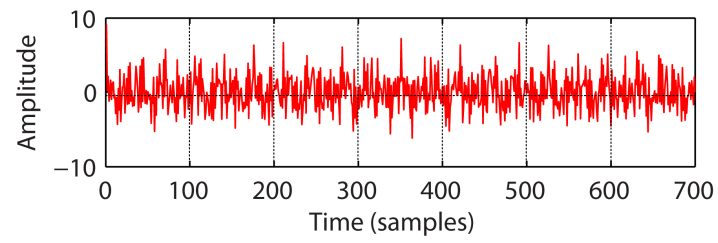

c)

Fig. 3 Time domain representations: a) Original clean signal, b) Original noisy signal, c) Reconstruction using L-estimate OMP algorithm
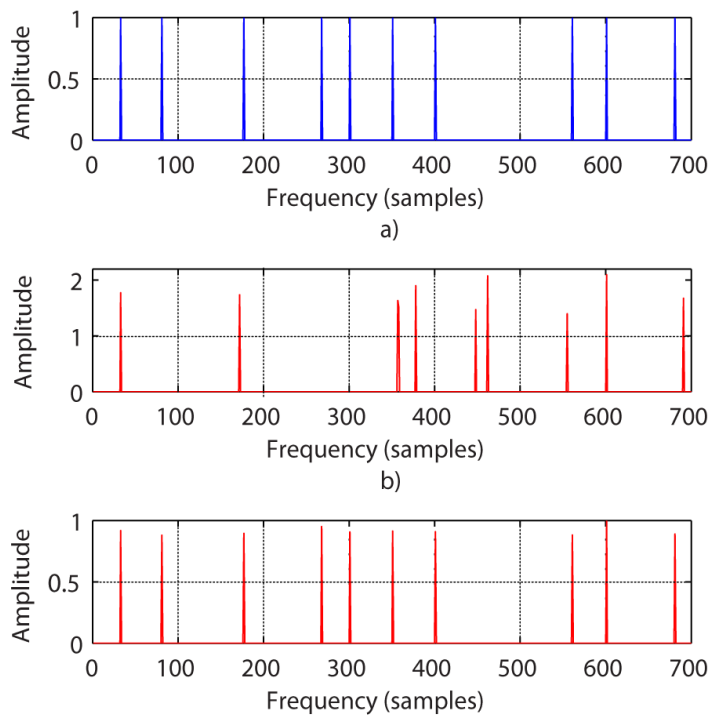

c)

Fig. 4. Fourier transform of: a) Original clean signal b) standard OMP reconstructed signal, c) Recovered signal using L-estimate OMP

Finally, we may conclude that the proposed algorithm is shown not to be sensitive to the choice of $M$. Namely, it is only important that the number of available samples after the L-estimation

$(M(1-2 \alpha)+4 \alpha)$ is sufficient for CS algorithm convergence. Generally, the procedure is efficient as long as $M(1-2 \alpha)+4 \alpha$ is above $20 \%$ of the total original signal length. Excessive number of missing samples, for example more than $85 \%$ of values, tends to significantly compromise performance. This topic has been intensively studied in [13], including the influence of the number of missing samples to the ability of CS reconstruction.

\section{CONCLUSION}

Sparse signal reconstruction methods based on the standard optimization algorithms (BP and OMP) combined with L-statistics are proposed. These methods retain all of the advantages of standard methods, while converging toward the correct signal recovery when dealing with additive impulse noise. This is achieved without introducing computationally complex constraints and minimization norms.

\section{REFERENCES}

1 R. Baraniuk, Compressive sensing, IEEE Signal Processing Magazine, 24(4), 2007, pp. 118-121

2 S. Stankovic, I. Orovic, E. Sejdic, Compressive sensing, Ch. 6 in Multimedia Signals and Systems, Springer, 2012.

3 F. Ahmad, and M.G. Amin, Through-the-wall human motion indication using sparsity-driven change detection, IEEE Trans. on Geoscience and Remote Sensing, 50(12), 2012, pp. 881-890 
4 P. Flandrin, and P. Borgnat, Time-Frequency Energy Distributions Meet Compressed Sensing, IEEE Transactions on Signal Processing, 58(6), 2010, pp. 2974-2982

5 E. Candes, and J. Romberg, L1-magic : Recovery of Sparse Signals via Convex Programming, http://www.acm.caltech.edu/11magic $\wedge \#$ code, 2005 , pp. $1-19$

6 J. A. Tropp, A. C. Gilbert, Signal Recovery From Random Measurements Via Orthogonal Matching Pursuit, IEEE Transaction on Information Theory, vol. 53, no.12, 2007

7 T. Zhang, Sparse Recovery with Orthogonal Matching Pursuit Under RIP, IEEE Transaction on Information Theory, 57(9), 2011, pp. 6215-6221

8 R. E. Carrillo, K. E. Barner, and T. C. Aysal, Robust sampling and reconstruction methods for sparse signals in the presence of impulsive noise, IEEE
Journal of Selected Topics in Signal Processing, 4(2), 2010, pp. 392-408,

9 J. L. Paredes and G.R. Arce, Compressive sensing signal reconstruction by weighted median regression estimates, IEEE Transactions on Signal Processing, 59(6), 2011, pp. 2585-2601

10 P.J. Huber, Robust Statistics, John Wiley\&Sons Inc., 1981.

11 I. Djurović, L. Stanković, and J. F. Böhme, Robust L-estimation based forms of signal transforms and time-frequency representations, IEEE Transactions on Signal Processing, 51(7), 2003, pp. 1753-1761

12 I. Pitas, A.N. Venetsanopoulos, Nonlinear digital filters: Principles and applications, Kluwer, 1990.

13 LJ. Stankovic, S. Stankovic, I. Orovic, M. Amin, Robust Time-Frequency Analysis based on the Lestimation and Compressive Sensing, IEEE Signal Processing Letters, 20(5), pp. 499-502 